\title{
Application of FRC in tunnel reinforcement
}

\author{
P. Procházka \\ Czech Technical University in Prague, Czech Republic
}

\begin{abstract}
Fibers in concrete should primarily restrain the material from cracks during the curing process and dehydration. For standard evaluation of the influence of the fibers, the three or four point test is studied, both experimentally and numerically on a straight beam. In tunnel lining the situation is slightly different. While the three or four point test leads to the assessment of the structure being only bended, in tunnel reinforcement not only this but also bending with normal force has to be observed. Moreover, the curing is affected by the non-uniform distribution of moistening, as from the side of the surrounding rock the measure of wet is principally higher than on the face where ventilation speeds up the dehydration. These two assumptions are considered and treated experimentally and numerically. Because a real tunnel lining is too large, a scale model is used and, consequently, more samples can be studied. Similarity rules and an appropriate calibration have to be obeyed. This paper is principally focused on the theory of possible coupled modeling of behavior of the one-sided moistened tunnel lining.
\end{abstract}

\section{Introduction}

Recently, the author published several papers on behavior of steel FRC with Dramix type fibers in underground structures, especially in tunnel linings, [1]. One-sided moistened concrete beams have been studied from the mechanical point of view. Scale models were prepared and studied.

The process, which we deal with in this paper, starts with coupled modeling targeted to description of one-sided moistened beams. Then arches are modeled and the mechanical properties are taken from the straight beam behavior. From the numerical point of view a linear system of equations for unconstrained problem has to be solved and when restrictions are imposed on stresses a nonlinear problem is to be observed. The solution of such a problem can be 
formulated in terms of series including eigenparameters, which simulate measure of different moistening at different layers in the structure. The application of the proposed problem is suitable for structures from composite materials, for laminated or layered structures, in tunnel construction, especially in NATM, etc. In order to approximate the real structure of tunnel lining, in our study a laminated hollow cylinder will be investigated from theoretical standpoint. Uniform distribution of eigenparameters is considered in each layer.

\section{Experiments with a beam}

The behavior of one-sided moistened FRC was studied on straight beam, since curvilinear beam could be modeled in a cumbersome way. The samples were prepared in an enameled basin, the tap water in which was pored, which was permanently added to hold the water level at a proper elevation. The wetted medium surrounding the sample simulated real situation on site. Consequently, the sample was prepared from two kinds of layers: the first layer was built up from high porously liquid-absorbing mass. This mass was separated from the concrete (the sample itself) by a filter paper to ensure convenient removal of the concrete slender element from the equipment for preparing the samples. As a consequence of this structure the lower side of the samples was permanently highly moistened. This was also the aim of the experimental study

The samples were built up by auxiliary timber frame; its thickness along three sides had the value of the right layer of the samples. The fourth side was free in the beginning of the layers creation. Then the first layer of the fiber reinforced concrete was constructed and the rectangular frame was completed by gluing the last part (the shape of it corresponded to the height of the layer). This stage of building the experimental equipment is depicted in Fig. 1, where also wire holders for sticking next molding are seen to create the following layers of the concrete. In this figure still no water is pored, and the three remaining moldings inside the form are still not yet put inside the form to create the first concrete layer.

In order to create the thicknesses of the layers as accurately as possible, the moldings were put into the form along all sides of the equipment, so that the room for the first and the subsequent layers was exactly defined. In other words, at each stage of layers creation, one planar frame was built up with the same thickness of moldings (as desired).

A plateau with the set up of fibers was drawn in Corel Draw on hardened paper of $200 \mathrm{~g} / \mathrm{m} 2$ In order to hold the cold temperature, which could cause temporary sticking of fibers to the paper, the temperature of the paper with the lay-up of fibers was dropped by putting the system in a freezer. Frozen at 32 degrees of Celsius every paper was three times sprinkled to assure the adhesion of fibers to the paper. The number of fibers was calculated in such a way that the fiber volume ratio was 1 percent. In each layer are 270 fibers in 11 interfaces of concrete layers (6 and 5 fibers are repeated in rows). The thicknesses of the layers are $6+4+\ldots+4+2 \mathrm{~mm}$. 
The concrete mixture was made of Knauf BP-8, the stones of which larger than $0.8 \mathrm{~mm}$ were removed by filtering.

For 28 days in room temperature the slender beam was dried in such a way that from the upper side a fan two times a day for 2 minutes was used from a distance of $2 \mathrm{~m}$. From below steady delivery of water was assured.

The final stage is seen in Fig. 2. The water was poured in the basin immediately after the first layer of concrete was put in the frame. The sample was not fully in a directly contact with the water, but along the interface of the sample with the plateau the moistening of the lower layer was ensured. The dimensions of the whole sample element were: $150 \times 250 \times 48 \mathrm{~mm}^{3}$. This sample was cut into beams of a cross section $2.8 \times 4.8 \mathrm{~mm}^{2}$, which were loaded by a concentrated load in the middle of span of the beams. The ratio of thickness to span was about $1 / 3$. From that point of view, the mechanical behavior was closer to a plate.

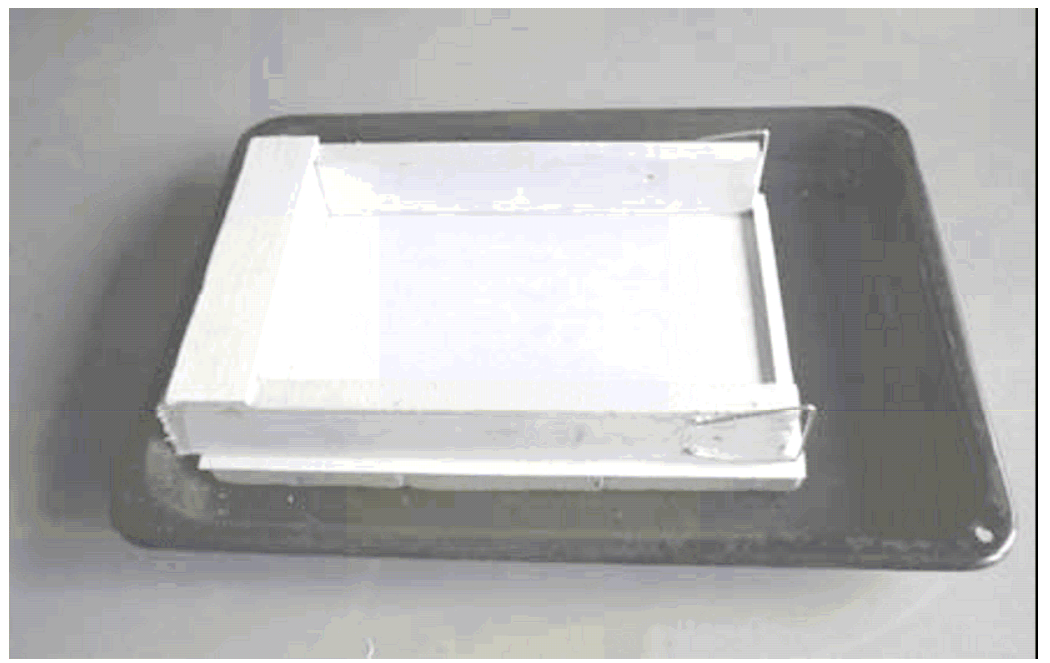

Figure 1: Experimental equipment with the basin and frame at the commencing stage.

Then a comparative study provided a time-dependent distribution of strains in the beam during the bending test. The one-sided moistened beam during the curing process and standard fiber reinforced beam are compared in both experimental studies, static and time dependent. Because a non-reinforced beam has very poor bearing capacity, it is not considered in these comparative studies.

A standard cement mixture has modulus of elasticity $\mathrm{E}=20 \mathrm{GPa}$ and Poisson's ratio $v=0.16$. Steel fibers possess modulus of elasticity $\mathrm{E}=197 \mathrm{GPa}$ and Poisson's ratio $v=0.3$. Regularly distributed Dramix-type fibers are used. A typical bending test with one concentrated load in the middle of $128 \mathrm{~mm}$ span of beams was applied. The beams were loaded on the moistened side to worsen the situation. It was expected that the pore pressure would expand the layers on the 
moistened side, while in the dry side the pore pressure slowly disappears. This caused a sort of prestress at the moistened side.

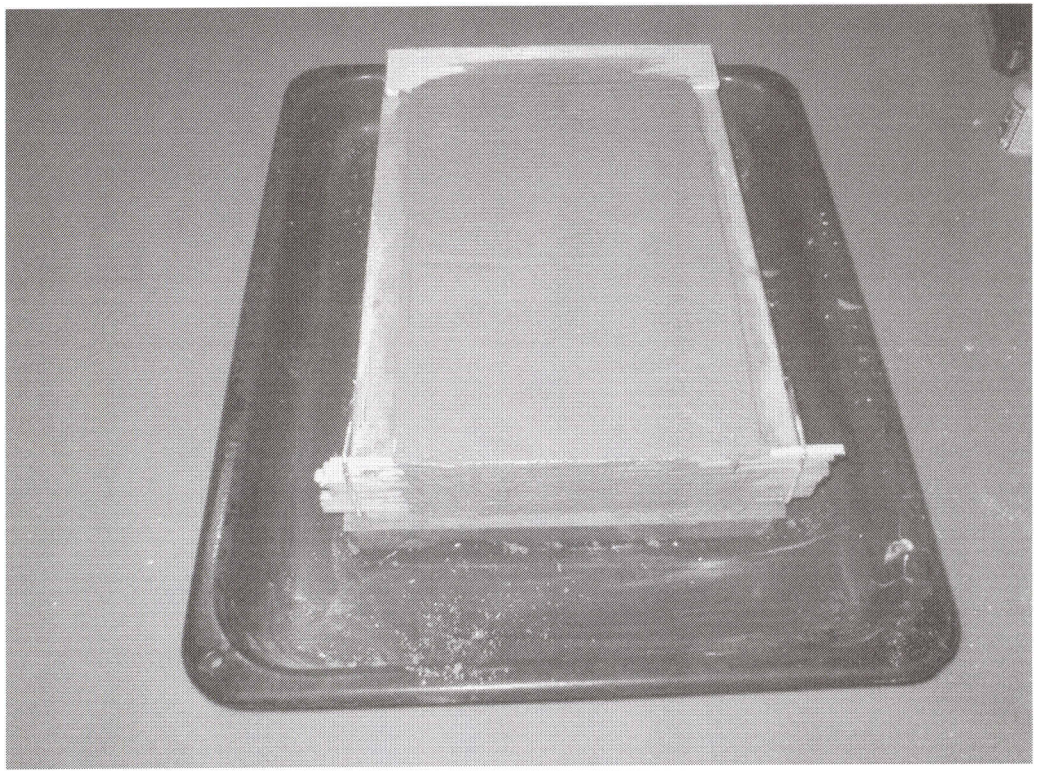

Figure 2: Final stage of the sample element after construction.

The aim of this study was to discover how the situation would worsen during the bending test comparing these samples with that without loading by the moistening.

In Fig. 3, a relation applied force - deflection is depicted. Two cases of the beams are compared: moistened and external moistening free plates are considered. Both types of beams were cured 28 days and then the experiments were accomplished.

From Fig. 3 one can see a sudden drop at the value of loading about $1.8 \mathrm{kN}$ in the case of moistened beam and about $2.6 \mathrm{kN}$ in the case of the wet-free beam. These drops differ also in deflections, which were in the first case $0.18 \mathrm{~mm}$ and $0.22 \mathrm{~mm}$ in the second case. Sudden opening of a crack below the concentrated load causes the drops of loading. After increase of loading peaks are reached. While the externally moistened beam attains $3.8 \mathrm{kN}$, the moistening free beam $4.5 \mathrm{kN}$. After this, the beams still bear, but their bearing capacity lowers and softening of the aggregate occurs. This is caused by a pulling of fibers out of the concrete matrix. When the destruction of the beams is attained, it can be seen that no steel fiber were disconnected, fibers were only pulled out of the concrete.

The different behaviors of both beams are caused by pore pressure, which is stored in cured beams. In mathematical models this property is very easy to describe. Pore pressure is a special case of eigenstrain tensor, $\mu$, or eigenstress tensor, $\lambda$, which both are expressed in the generalized Hooke's law as: 


$$
\sigma=L(\varepsilon-\mu) \text { or } \sigma=L \varepsilon+\lambda
$$

where $\sigma$ is the stress tensor and $\varepsilon$ is the strain tensor. $L$ is the elastic material stiffness matrix.

Another type of experiment was carried out on these beams. A time dependent behavior of both types of beams is observed by bending with the same disposition as in the previous experiments, see Fig. 4.

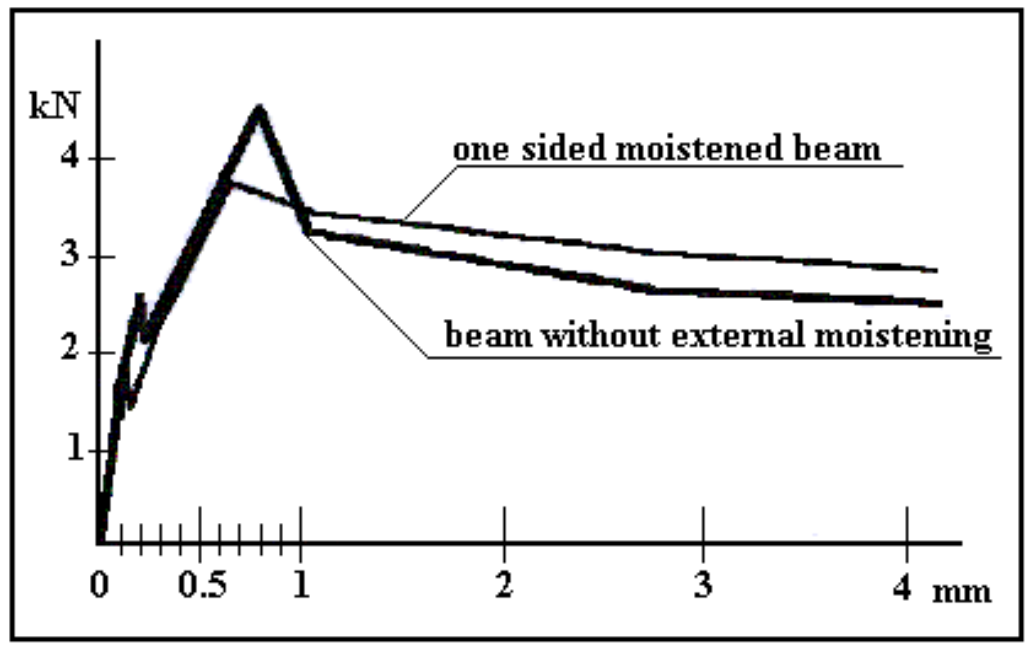

Figure 3: Deflection - concentrated load relation.

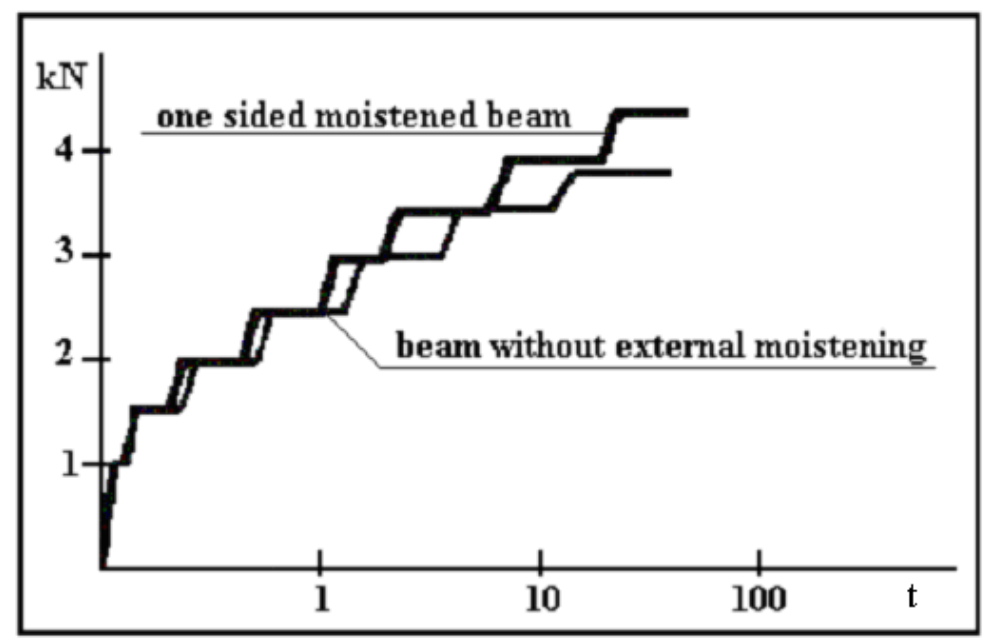

Figure 4: $\quad$ Time dependent behavior of the beams (creep). 
From the picture it is seen that the behavior of both types of beams is not different that much. Only in the neighborhood of the peak values the differences are larger than for the lesser values of the concentrated load.

From both examples we have a conclusion that both bearing capacity and time dependent deflections are larger in moistened beams. Nevertheless, the differences are not that much decisive that the steel fiber reinforced concrete has to be removed from the production for the type of structures, such as underground works (tunnels, retaining walls), foundations, floor decking, etc.

\section{Numerical description of a cylindrical segment}

In this section we concentrate our attention on a special type of one-sided moistened FRC structure, which can be used in design of underground works, foundation engineering, floor decking of industry halls, sport halls, etc. The mechanical behavior of the moistened arches was compared with the fiberreinforced straight beams. The reinforcement of both types of beams was put into structures regularly (uniformly) in a very careful way, as described in the above text.

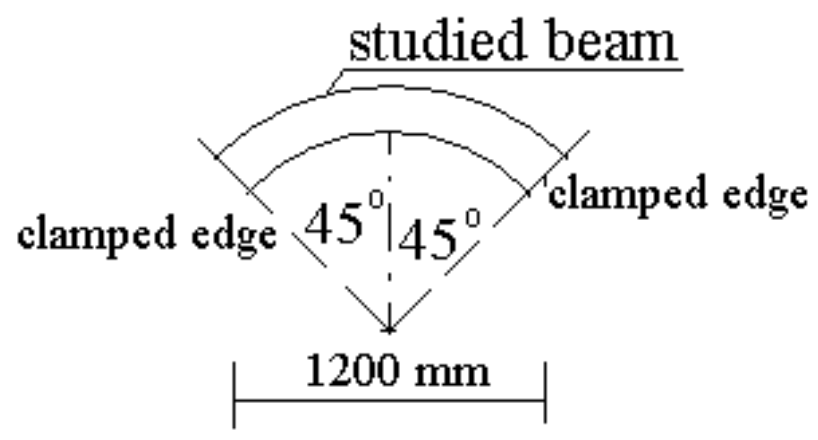

Figure 5: Geometry and disposition of the beam under study.

It was shown that the bearing capacity of the moistened structure is less than that of beams created in the standard way. Also the creep seems to be more important and larger in the case of moistened structure.

\section{Relations on a single layer}

The clamped arch can be solved by Fourier method: the unknowns and the loading are split into series in one direction and in the remaining direction the ordinary differential equation is solved. The problem of clamped arch is divided into solution of simply supported arch and the rotation at clamped edges is annihilated by applying external bending moment. The solution of the latter case can be found in [2]. The first problem is a generalization of the problem from [3]. Results from similar cases can be seen in [4]. 
Introducing cylindrical coordinate system Or $\varphi z$, components of the displacement vector are considered: $u=u_{r}, v=u_{\varphi}, w=u_{z}$, where $u_{r}$ is the displacement in the radial direction and $u_{\varphi}$ is the displacement in the circumferential (hoop) direction and $w=u_{z}$ is the displacement in the axial direction. The cylindrically orthotropic structure is a particular example of a cylindrically anisotropic solid with the constitutive equations written as (in this section the indices describing number of the layer are omitted):

$$
\left(\begin{array}{c}
\sigma_{r} \\
\sigma_{\varphi} \\
\sigma_{z} \\
\sigma_{r \varphi} \\
\sigma_{\varphi z} \\
\sigma_{r z}
\end{array}\right)=\left[\begin{array}{cccccc}
L_{r r} & L_{r \varphi} & L_{r z} & 0 & 0 & 0 \\
L_{r \varphi} & L_{\varphi \varphi} & L_{\varphi z} & 0 & 0 & 0 \\
L_{r z} & L_{\varphi z} & L_{z z} & 0 & 0 & 0 \\
0 & 0 & 0 & G_{r \varphi} & 0 & 0 \\
0 & 0 & 0 & 0 & G_{\varphi z} & 0 \\
0 & 0 & 0 & 0 & 0 & G_{r z}
\end{array}\right]\left(\begin{array}{c}
\varepsilon_{r}-\mu_{r} \\
\varepsilon_{\varphi r}-\mu_{\varphi r} \\
\varepsilon_{z r}-\mu_{z r} \\
2\left(\varepsilon_{r \varphi}-\mu_{r \varphi}\right) \\
2\left(\varepsilon_{\varphi z}-\mu_{\varphi z}\right) \\
2\left(\varepsilon_{r z}-\mu_{r z}\right)
\end{array}\right)
$$

where the stress tensor $\boldsymbol{\sigma}$ was related with the strain tensor $\boldsymbol{\varepsilon}$ and the eigenstrain tensor $\boldsymbol{\mu}$ through the material stiffness matrix $\mathbf{L}$, and $L_{i j}, G_{r \varphi}, G_{\varphi z}, G_{r z}$ are the stiffness coefficients. Nine stiffness coefficients and six eigenstrains describe this kind of anisotropy. Cylindrical orthotropy is characterized by the fact that properties in the tangential, radial and axial directions are distinct; in other words, the material is orthotropic in a Cartesian system which is located at any point within the structure, with the three axes pointing in the axial, tangential and radial directions respectively. If $L_{r r}>L_{\varphi \varphi}$ then the material is called radially orthotropic, and if $L_{\varphi \varphi}>L_{r r}$ then it is called circumferentially orthotropic.

In the special case of transversally isotropic solid which may represent the average structure of fiber reinforced material, nine independent material constants in (1) are related as:

$$
L_{r r}=L_{\varphi \varphi}, \quad L_{r z}=L_{\varphi z}, \quad G_{r z}=G_{\varphi z}, \quad L_{r r}-L_{r \varphi}=2 G_{r \varphi}
$$

In what follows we concentrate our attention on 2D case, assuming a long enough cylinder. Then the number of displacement components is reduced to $u=u_{r}, v=u_{\varphi}, w=u_{z}$, and three stresses remain non-zero: $\sigma_{r}, \sigma_{\varphi}, \tau_{r \varphi}$. The cylindrical coordinate system reduces to polar one.

Recall basic geometrical relations:

$$
\varepsilon_{r}=\frac{\partial u}{\partial r}, \quad \varepsilon_{\varphi}=\frac{1}{r} \frac{\partial v}{\partial \varphi}+\frac{u}{r}, \quad \varepsilon_{\mathrm{r} \varphi}=\frac{1}{r} \frac{\partial u}{\partial \varphi}+\frac{\partial v}{\partial r}-\frac{v}{r}
$$

If we adopt the assumption $\sigma_{z}=0$ and isotropic material is considered, Hooke's law takes the form: 


$$
\varepsilon_{r}-\mu_{r}=\frac{1}{E}\left(\sigma_{r}-v \sigma_{\varphi}\right), \quad \varepsilon_{\varphi}-\mu_{\varphi}=\frac{1}{E}\left(\sigma_{\varphi}-v \sigma_{r}\right), \quad \varepsilon_{r \varphi}-\mu_{r \varphi}=\frac{1}{G} \sigma_{r \varphi}
$$

where $v$ is Poisson's ratio. Substituting the last equations in the geometrical equations yields the inverse relations of Hooke's law for expressing stresses in the terms of displacements:

$$
\begin{gathered}
\sigma_{r}=L_{r r}\left(\varepsilon_{r}-\mu_{r}\right)+L_{r \varphi}\left(\varepsilon_{\varphi}-\mu_{\varphi}\right)=L_{r r}\left(\frac{\partial u}{\partial r}-\mu_{r}\right)+L_{r \varphi}\left(\frac{1}{r} \frac{\partial v}{\partial \varphi}+\frac{u}{r}-\mu_{\varphi}\right) \\
\sigma_{\varphi}=L_{r \varphi}\left(\frac{1}{r} \frac{\partial v}{\partial \varphi}+\frac{u}{r}-\mu_{\varphi}\right)+L_{\varphi \varphi}\left(\frac{\partial u}{\partial r}-\mu_{r}\right) \\
\sigma_{r \varphi}=G\left(\varepsilon_{r \varphi}-\mu_{r \varphi}\right)=G\left(\frac{1}{r} \frac{\partial u}{\partial \varphi}+\frac{\partial v}{\partial r}-\frac{v}{r}-\mu_{r \varphi}\right)
\end{gathered}
$$

Two equations of equilibrium provide relations among three components of stress tensor:

$$
\frac{\partial \sigma_{r}}{\partial r}+\frac{1}{r}\left(\frac{\partial \sigma_{r \varphi}}{\partial \varphi}+\sigma_{r}-\sigma_{\varphi}\right)=0, \quad \frac{\partial \sigma_{r \varphi}}{\partial r}+\frac{1}{r}\left(\frac{\partial \sigma_{\varphi}}{\partial \varphi}+2 \sigma_{r \varphi}\right)=0
$$

Expanding two components of displacements into sine and cosine series and denoting the $k$-th term by $U_{r}^{(k)}$ and $U_{\varphi}^{(k)}$ yields

$$
\begin{aligned}
& u_{r}^{(k)}(r, \varphi)=U_{r}^{(k)}(r) \cos 2 k \varphi, \\
& u_{\varphi}^{(k)}(r, \varphi)=U_{\varphi}^{(k)}(r) \sin 2 k \varphi, \\
& u_{z}^{(k)}=0
\end{aligned}
$$

where $U_{r}^{(k)} \equiv U^{(k)}$ and $U_{\varphi}^{(k)} \equiv V^{(k)}$ are unknown functions of $r$ which need to be determined from the equations of equilibrium. In particular, the substitution of (1) in the stress-strain relations gives stresses which, when substituted into the equations of equilibrium in cylindrical coordinates, provide the following equations for evaluation of $U^{(k)}$ and $V^{(k)}$.

Similarly the eigenparameters will be expanded into series with the $k$-th components:

$$
\begin{gathered}
\mu_{r}^{(k)}(r, \varphi)=R^{(k)}(r) \cos 2 k \varphi, \quad \mu_{\phi}^{(k)}(r, \phi)=F^{(k)}(r) \cos 2 k \varphi, \\
\mu_{r \phi}^{(k)}(r, \varphi)=M^{(k)}(r) \sin 2 k \varphi
\end{gathered}
$$


Components of strains, (2), and stresses, (6), are then expressed as (identification $(k)$ of the number in the series is dropped out and the prime denotes derivative by $r$ ):

$$
\begin{gathered}
\varepsilon_{r}^{(k)}=U^{\prime} \cos 2 k \varphi, \quad \varepsilon_{\varphi}^{(k)}=\frac{1}{r}(2 k V+U) \cos 2 k \varphi, \quad \varepsilon_{r \varphi}^{(k)}=\left(-\frac{2 k}{r} U+V^{\prime}-\frac{1}{r} V\right) \sin 2 k \varphi \\
\sigma_{r}^{(k)}=\left[L_{r r}\left(U^{\prime}-R\right)+L_{r \varphi} \frac{1}{r}(2 k V+U-F)\right] \cos 2 \varphi \\
\sigma_{\varphi}^{(k)}=\left[L_{r \varphi} \frac{1}{r}(2 k V+U-F)+L_{\varphi}\left(U^{\prime}-R\right)\right] \cos 2 \varphi \\
\sigma_{r \phi}^{(k)}=\mathrm{G}\left[-\frac{2 k}{r} U+V^{\prime}-\frac{1}{r} V-M\right] \sin 2 \phi
\end{gathered}
$$

Substituting (10), (11) to (6) and using the last four relations, two equations for unknown amplitudes of displacements read as:

$$
\begin{gathered}
U^{\prime \prime}-R^{\prime \prime}+(1-v)\left(U^{\prime}-R^{\prime}\right)+\frac{v}{r}\left(2 k V^{\prime}+U^{\prime}-F^{\prime}\right)+ \\
+\frac{2 k(1-v)}{r}\left(-\frac{2 k}{r} U+V^{\prime}-\frac{V}{r}-M\right)-\frac{1}{r}[2 k V+U-F]=0 \\
V^{\prime \prime}-\frac{3}{r} V^{\prime}+\frac{8 k^{2}+v-1}{1-v} V-\frac{4 k+v}{r} U^{\prime}+\frac{4 k(v-3)}{(1-v) r^{2}} U-M^{\prime}+\frac{M}{r}+\frac{v}{R} R^{\prime}+\frac{F}{r^{2}}=0
\end{gathered}
$$

The last two equations lead to the solution, which defines the displacements on each lamina. Four integration constants are determined from interfacial conditions between adjacent layers. The angle determined from the above approach is eliminated by introducing moments at the end points to get conditions for clamped segment. The influence of such a moment to the segment is taken from [1], where the approach is described in details.

\section{Conclusions}

In this paper the procedure for calculating a segment of one-sided moistened tunnel lining from FRC. Information from straight one-sided moistened beams has been transferred to the segment and then the numerical procedure is suggested. Since it allows us to apply semianalytical (Fourier) solution, the procedure is relatively easy. First simply supported segment is considered, then using the approach from [2] for involvement of given moment at the end points, the clamped edge can be simulated. 
400 High Performance Structures and Materials III

\section{Acknowledgment}

This paper was prepared under financial support of GA AV ČR, project No. IAA 2119402.

\section{References}

[1] Procházka, P., Starikov, N.: Optimal Slope of Dramix Type Fibers in Reinforced Concrete, 6th World Congresses of Structural and Multidisciplinary Optimization, Rio de Janeiro, 30 May - 03 June 2005, Brazil.

[2] Lekhnitski, S.G. Anisotropic plates. Gordon and Breach Sci. Publ. NYC London - Paris, 1968.

[3] Chen, T., Dvorak, G.J. and Benveniste, Y. Stress fields in composites reinforced by coated cylindrically orthotropic fibers. Mechanics of Materials 9 (1990) 17-32.

[4] Sokolnikoff, I.S. Mathematical theory of elasticity. $2^{\text {nd }}$ edition, McGrawHill, New York, 1996. 